\title{
Enade em Odontologia: análise e reflexões à luz da Taxonomia de Bloom Revisada
}

\author{
Suzely Adas Saliba Moimaz; Marcelo Augusto Amaral**; Cléa Adas Saliba Garbin*; Nemre Adas \\ Saliba*
}

* Professora Titular do Departamento de Odontologia Infantil e Social, Faculdade de Odontologia de Araçatuba, Universidade Estadual Paulista

** Aluno de Doutorado do Programa de Pós-Graduação em Odontologia Preventiva e Social, Faculdade de Odontologia de Araçatuba, Universidade Estadual Paulista

Recebido em 23/01/2017. Aprovado em 17/07/2017.

\section{RESUMO}

Objetivou-se analisar as questões de Saúde Coletiva (SC) das edições do Exame Nacional de Desempenho dos Estudantes (Enade) de Odontologia e o desempenho dos concluintes da Universidade Estadual Paulista (Unesp), Faculdade de Odontologia de Araçatuba (FOA). Realizouse uma pesquisa documental, com análise das avaliações de 2004, 2007, 2010, 2013. Para a identificação das questões de SC presentes nos exames foram utilizados os objetos elencados no Artigo 86 da Consolidação das Normas para Procedimentos nos Conselhos de Odontologia, e classificadas segundo a Taxonomia de Bloom Revisada (TBR): "Lembrar, Entender, Aplicar, Analisar, Avaliar, Criar". Foram coletados os índices de desempenho dos concluintes da Unesp/FOA nos relatórios referentes às quatro avaliações. Foram consideradas 28 questões, sendo 23 objetivas e cinco discursivas. As dimensões "Lembrar", "Entender" e "Aplicar" da TBR foram verificadas em sete questões e a média de acertos dos egressos para estas dimensões foi $65,53 \%$. A dimensão “Analisar" apresentou-se em uma questão em 2004, quatro em 2007, quatro em 2010 e duas questões na edição de 2013 e o aproveitamento médio foi de 44,84\%. Das dez questões da dimensão "Avaliar", o desempenho médio foi de $61,00 \%$ na edição de 2010 , com acerto variando de $43,10 \%$ a $81,40 \%$, e para a edição de 2013 a média foi de $60,60 \%$, com variação de $32,30 \%$ a 79,20\%. Os objetivos educacionais "Analisar" e "Avaliar" foram mais prevalentes nas edições do Exame Nacional de Desempenho dos Estudantes e as questões classificadas nestas dimensões de maior nível cognitivo apresentaram menor taxa de acertos, quando comparadas às dimensões "Lembrar", "Entender" e "Aplicar".

Descritores: Avaliação Educacional. Classificação. Ensino. Odontologia.

\section{INTRODUÇÃO}

A avaliação da educação superior no Brasil tem destaque dentre as políticas educacionais desde meados da década de 1990, com a criação de avaliações periódicas das instituições e cursos superiores e tornou-se obrigatória para todos os concluintes a partir de $1996^{1}$.
Inicialmente foi regulamentada por meio do Exame Nacional de Cursos (ENC), também conhecido como Provão e que contou com oito aplicações anuais durante o período de 1996 a $2003^{2}$. O ENC trouxe importantes elementos para a reflexão e identificação de distorções dos Projetos Pedagógicos dos Cursos e sua execução, 
visando à melhoria e qualidade do curso, e consequentemente, o alcance de melhor desempenho neste exame ${ }^{3}$.

Posteriormente surge o Sistema Nacional de Avaliação da Educação Superior e o Exame Nacional de Desempenho dos Estudantes (Enade), que responde atualmente pela avaliação dos estudantes da educação superior. Este exame é aplicado trienalmente e se propõe a verificar várias dimensões de competências e habilidades profissionais em um processo avaliativo mais abrangente. A prova é composta por dez questões de formação geral (objetivas e discursivas) e trinta questões de componente específico (objetivas e discursivas) $^{4}$.

Uma década depois, surge o Plano Nacional de Educação (PNE), como um instrumento de planejamento do Estado democrático de direito que orienta a execução e o aprimoramento de políticas públicas do setor. O PNE 2014-2024 trouxe diversas diretrizes, entre elas a qualidade da educação superior, que visa aperfeiçoar o Enade, fomentando a melhoria dos resultados de aprendizagem, de modo que, em cinco anos, pelo menos $60 \%$ dos estudantes apresentem desempenho positivo igual ou superior a $60 \%$ neste exame ${ }^{5}$.

Neste contexto, a avaliação deve ser uma prática constante na educação superior e um dos instrumentos existentes que pode vir a facilitar esse processo é a taxonomia proposta por Bloom; Hastings; Madaus ${ }^{6}$, que tem, explicitamente, como objetivo ajudar no planejamento, organização e controle dos objetivos de aprendizagem.

A taxonomia dos objetivos cognitivos de Bloom, nomenclatura mais conhecida, divide-se em três domínios: cognitivo, afetivo e psicomotor. O domínio cognitivo refere-se à memória e ao desenvolvimento de habilidades intelectuais, o afetivo, avalia o interesse, atitudes e valores no processo educacional, e o psicomotor compreende as habilidades e competências motoras ${ }^{6}$.

A Taxonomia de Bloom do domínio cognitivo é estruturada em níveis de complexidade crescente, do mais simples ao mais complexo, e isso significa que, para adquirir uma nova habilidade pertencente ao próximo nível, o aluno deve ter dominado e adquirido a habilidade de nível anterior ${ }^{7}$.

A partir das novas tecnologias incorporadas ao sistema educacional, foi observada a necessidade de adaptações e elaboração de uma revisão da taxonomia original, dando origem a Taxonomia de Bloom Revisada (TBR), que passou a estabelecer novos objetivos educacionais ${ }^{7}$.

A TBR vem sendo adotada nas últimas décadas como base para a elaboração e estudos de instrumentos de avaliação, assim como o desenvolvimento de currículos acadêmicos. As mudanças propostas envolveram alterações na terminologia inicialmente utilizada, onde os substantivos "Conhecimento, Compreensão, Aplicação, Análise, Síntese e Avaliação" foram substituídos por "Lembrar, Entender, Aplicar, Analisar, Avaliar e Criar". A escolha da TBR para esta pesquisa deve-se também ao fato de que ela pode ser aplicada a diferentes áreas do conhecimento, uma vez que suas novas categorias permitem classificação de diferentes conteúdos educacionais ${ }^{9}$.

É importante que as questões ou itens das avaliações sejam analisadas para orientar e subsidiar os formuladores dos exames nacionais, bem como, educadores, gestores educacionais e instituições de ensino superior na elaboração de avaliações que melhorem o desempenho acadêmico e o processo de ensino-aprendizagem na área de Odontologia.

$\mathrm{Na}$ presente pesquisa objetivou-se analisar as questões de Saúde Coletiva das edições do Exame Nacional de Desempenho dos Estudantes 
de Odontologia de 2004 a 2013, utilizando a Taxonomia de Bloom Revisada e o desempenho dos concluintes da Universidade Estadual Paulista, Faculdade de Odontologia de Araçatuba.

\section{MATERIAIS E MÉTODOS}

Realizou-se uma pesquisa documental de todas as edições do Enade para o curso de Odontologia, desde a sua instituição, em 2003, até o ano de 2013. Foram analisadas as edições das avaliações de 2004, 2007, 2010, 2013 por meio de uma consulta no portal do Instituto Nacional de Estudos e Pesquisas Educacionais Anísio Teixeira (INEP) ${ }^{10}$.

Para a identificação das questões objetivas e discursivas de Saúde Coletiva (SC) presentes nos exames, foram utilizados os objetos elencados no Artigo 86 da Consolidação das Normas para Procedimentos nos Conselhos de Odontologia (Resolução 63) referentes a especialidade de SC e apresentados a seguir ${ }^{11}$ :

a) análise socioepidemiológica dos problemas de saúde bucal da comunidade;

b) elaboração e execução de projetos, programas e outros sistemas de ação coletiva ou de saúde pública visando a promoção, o reestabelecimento e o controle da saúde bucal; e,

c) participar, em nível administrativooperacional de equipe multiprofissional, por intermédio de:

1. organização de serviços;

2. gerenciamento em diferentes setores e níveis de administração em saúde pública;

3. vigilância sanitária;

4. controle das doenças; e

5. educação em saúde pública.

As avaliações do Enade de 2004 e 2007 apresentaram 26 questões objetivas e quatro discursivas. Em 2010 e 2013, houve 27 questões objetivas e três discursivas e em todas as edições o número de questões específicas totalizou 30 itens.

Posteriormente à identificação das questões que abordavam os objetos de conhecimento de Saúde Coletiva presentes nos exames $(n=28)$, realizou-se a classificação segundo a Taxonomia de Bloom Revisada ${ }^{7}$ por meio das dimensões dos processos cognitivos: "Lembrar, Entender, Aplicar, Analisar, Avaliar, Criar" apresentadas no quadro 1.

Para análise segundo a TBR, cada questão do Enade foi avaliada e classificada segundo a dimensão do processo cognitivo, levando-se em consideração os seguintes critérios: nível de abstração, extensão e profundidade dos objetivos educacionais requeridos pela taxonomia em questão. A seguir foi elaborada um quadro para apresentar a distribuição das questões por categorias e o processo cognitivo utilizado para a aquisição do conhecimento em SC nos respectivos exames, a partir dos comandos verbais apresentados nos enunciados.

O desempenho dos estudantes da Universidade Estadual Paulista (Unesp), Faculdade de Odontologia de Araçatuba (FOA) nas questões da área de Saúde Coletiva foi analisado com base nos relatórios de cursos e institucionais do INEP ${ }^{12-15}$.

Após verificação dos percentuais de acertos dos concluintes da instituição pesquisada a partir da TBR foi realizada análise estatística com auxílio do software Bioestat 5.3. Em seguida, os dados de 2010 foram submetidos ao teste ANOVA, com nível de significância de 5\%. Por não apresentarem distribuição paramétrica, os dados de 2004, 2007, 2013 da Unesp/FOA, Regional e Brasil foram submetidos ao teste de Kruskal-Wallis $(\mathrm{p}<0,05)$.

Por se tratar de pesquisa com dados secundários públicos dispensa-se a avaliação por Comitê de Ética em Pesquisa. 
Quadro 1. Estrutura do processo segundo taxonomia de Bloom revisada.

1. Lembrar: relacionado a reconhecer e reproduzir ideias e conteúdos.

2. Entender: relacionado a estabelecer uma conexão entre o novo e o conhecimento prévio adquirido.

3. Aplicar: relacionado a executar ou usar um procedimento em uma situação específica, ou uma situação nova.

4. Analisar: relacionado a dividir a informação em partes relevantes e irrelevantes, importantes e menos importantes e entender a inter-relação entre as partes

5. Avaliar: relacionado a realizar julgamentos baseados em critérios e padrões qualitativos e quantitativos ou de eficiência e eficácia.

6. Criar: relacionado ao desenvolvimento de ideias novas e originais, produtos e métodos por meio da percepção da interdisciplinaridade e interdependência de conceitos.

Fonte: Ferraz, Belhot ${ }^{7}$.

\section{RESULTADOS E DISCUSSÃO}

$\mathrm{Na}$ análise das quatro avaliações aplicadas desde a instituição do Enade até 2013, foram consideradas 28 questões da área de SC, sendo 23 objetivas e cinco discursivas. Observou-se aumento significativo do número de questões da área de SC ao longo dos anos ${ }^{1}$. Em 2004, a área apresentou cinco questões objetivas (5/26 - 16,66\%), sendo as questões 09, 10, 11 e 12 objetivas e uma discursiva (03). No Enade de 2007 o conteúdo de SC apresentou sete questões (7/26 - 23,33\%), sendo as questões 19, 31, 32, 33 e 34 objetivas e duas discursivas (39 e 40).

Nas edições de 2010 e 2013 oito questões (8/27 - 26,66\%) contemplaram os conteúdos de $\mathrm{SC}$, sendo uma única questão discursiva para cada uma destas últimas edições avaliadas (1/3).

Considerando as quatro edições do Enade avaliadas, as dimensões "Lembrar, Entender e Aplicar" da Taxonomia de Bloom Revisada foram verificadas em uma, três e três questões, respectivamente, totalizando sete questões e envolvendo conteúdos de análise socioepidemiológica dos problemas de saúde bucal da comunidade e elaboração e execução de projetos, programas e outros sistemas de ação coletiva ou de saúde pública visando a promoção, o reestabelecimento e o controle da saúde bucal (quadro 2).

Verifica-se ainda no quadro 2, um incremento de questões de maior nível de cognição ao longo dos anos dos Enade avaliados.

A tabela 1 apresenta os valores percentuais de acertos dos concluintes da Unesp/FOA na parte do componente específico avaliado. Os percentuais de acerto estão colocados em ordem crescente, ao longo das edições, permitindo a comparação das médias obtidas por estes estudantes com os da mesma região e os resultados nacionais.

Comparando-se os resultados das edições do Enade avaliadas, constata-se que houve melhor desempenho nas questões objetivas, tanto entre concluintes da Unesp/FOA ou Região ou Brasil. Nas questões discursivas, verificou-se um desempenho inferior, entre concluintes das três categorias analisadas em algumas questões dos exames avaliados (tabela 1). 
Quadro 2. Classificação das questões de Saúde Coletiva, nas edições do Enade em Odontologia de acordo com a TBR $(\mathrm{n}=28)$.

\begin{tabular}{|c|c|c|c|c|c|c|c|}
\hline \multirow[b]{2}{*}{ Enade } & \multicolumn{7}{|c|}{ Questões de Odontologia } \\
\hline & 1. Lembrar & 2. Entender & 3. Aplicar & 4. Analisar & 5. Avaliar & 6. Criar & Total \\
\hline 2004 & 10 & 9,11 & 12 & $\mathrm{D}(03)$ & & & 5 \\
\hline 2007 & & 33 & $\begin{array}{c}34 \\
D(39)\end{array}$ & $\begin{array}{c}19,31,32 \\
\mathrm{D}(40)\end{array}$ & & & 7 \\
\hline 2010 & & & & $\begin{array}{c}13,34,35 \\
D(40)\end{array}$ & $16,20,31,37$ & & 8 \\
\hline 2013 & & & & 24,28 & $\begin{array}{c}11,14,25,26,31 \\
\mathrm{D}(05)\end{array}$ & & 8 \\
\hline Total & 1 & 3 & 3 & 11 & 10 & 0 & 28 \\
\hline
\end{tabular}

D: Questão discursiva.

Tabela 1. Percentual de acertos dos concluintes da Unesp/FOA, Região e Brasil, no componente de conhecimento específico da área de Saúde Coletiva nas edições do Enade avaliadas.

\begin{tabular}{|c|c|c|c|c|c|}
\hline Ano & Questão & Dimensão & FOA (\%) & Região (\%) & Brasil (\%) \\
\hline 2004 & 03 & Analisar & 21,7 & 20,3 & 22,1 \\
\hline 2004 & 09 & Entender & 12,1 & 23,0 & 26,0 \\
\hline 2004 & 10 & Lembrar & 75,8 & 58,9 & 59,5 \\
\hline 2004 & 11 & Entender & 93,9 & 89,1 & 89,7 \\
\hline 2004 & 12 & Aplicar & $*$ & $*$ & $*$ \\
\hline 2007 & 19 & Analisar & 85,7 & 69,4 & 70,1 \\
\hline 2007 & 31 & Analisar & 35,7 & 30,0 & 31,4 \\
\hline 2007 & 32 & Analisar & 71,4 & 36,7 & 38,7 \\
\hline 2007 & 33 & Entender & 64,3 & 43,3 & 44,0 \\
\hline 2007 & 34 & Aplicar & 42,9 & 37,3 & 38,6 \\
\hline 2007 & 39 & Aplicar & 81,4 & 81,1 & 81,6 \\
\hline 2007 & 40 & Analisar & 48,2 & 39,5 & 39,9 \\
\hline 2010 & 13 & Analisar & 78,4 & 54,5 & 49,8 \\
\hline 2010 & 16 & Avaliar & 50,0 & 51,3 & 51,9 \\
\hline 2010 & 20 & Avaliar & 69,6 & 55,4 & 56,3 \\
\hline 2010 & 31 & Avaliar & 43,1 & 49,6 & 52,1 \\
\hline 2010 & 34 & Analisar & $*$ & $*$ & $*$ \\
\hline 2010 & 35 & Analisar & 38,2 & 28,3 & 32,4 \\
\hline 2010 & 37 & Avaliar & 81,4 & 78,2 & 80,0 \\
\hline 2010 & 40 & Analisar & $*$ & $*$ & $*$ \\
\hline 2013 & 05 & Avaliar & 77,9 & 72,4 & 73,6 \\
\hline 2013 & 11 & Avaliar & 79,2 & 65,1 & 70,0 \\
\hline 2013 & 14 & Avaliar & 32,3 & 24,6 & 29,1 \\
\hline 2013 & 24 & Analisar & 45,8 & 36,9 & 38,7 \\
\hline 2013 & 25 & Avaliar & $*$ & $*$ & $*$ \\
\hline 2013 & 26 & Avaliar & 52,1 & 47,7 & 47,4 \\
\hline 2013 & 28 & Analisar & 32,3 & 31,2 & 34,3 \\
\hline 2013 & 31 & Avaliar & 61,5 & 50,2 & 53,7 \\
\hline
\end{tabular}

*: Questão anulada.

Observa-se que, tanto na parte objetiva obtiveram desempenhos superiores ao dos quanto na discursiva do componente específico estudantes da Região e Brasil, no entanto, não analisado, em todas as edições avaliadas do houve diferença estatisticamente significativa Enade, os estudantes concluintes da Unesp/FOA (tabela 2). Analisando-se os resultados de todas 
as edições avaliadas dos estudantes da Unesp/FOA, verificou-se que a média geral de acertos da área de Saúde Coletiva foi de 56,70\%. Os desempenhos médios entre concluintes da Região foi 48,94\% e do Brasil 50,47\% (gráfico 1).
A média de acertos dos estudantes egressos da Unesp/FOA para estas três primeiras dimensões foi $65,53 \%$. A dimensão "Lembrar" apresentou uma única questão no Enade de 2004 e com 75,80\% de acerto (gráfico 2).

Tabela 2. Distribuição das medianas, média e valores de p segundo o desempenho da Unesp/FOA, Região e Brasil nas questões validadas das edições do Enade 2004-2013 (n=24).

\begin{tabular}{c|c|c|c|c}
\hline & $\begin{array}{c}\mathbf{2 0 0 4} \\
\text { Mediana }\end{array}$ & $\begin{array}{c}\mathbf{2 0 0 7} \\
\text { Mediana }\end{array}$ & $\begin{array}{c}\mathbf{2 0 1 0} \\
\text { Média }\end{array}$ & $\begin{array}{c}\mathbf{2 0 1 3} \\
\text { Mediana }\end{array}$ \\
\hline FOA & 48,75 & 64,30 & 60,11 & 52,10 \\
Região & 40,95 & 39,50 & 52,88 & 47,70 \\
Brasil & 42,75 & 39,90 & 53,75 & 52,10 \\
\hline Valor de p & $0,91^{*}$ & $0,30^{*}$ & $0,72^{* *}$ & $0,66^{*}$ \\
\hline
\end{tabular}

* teste Kruskal-Wallis ** teste ANOVA

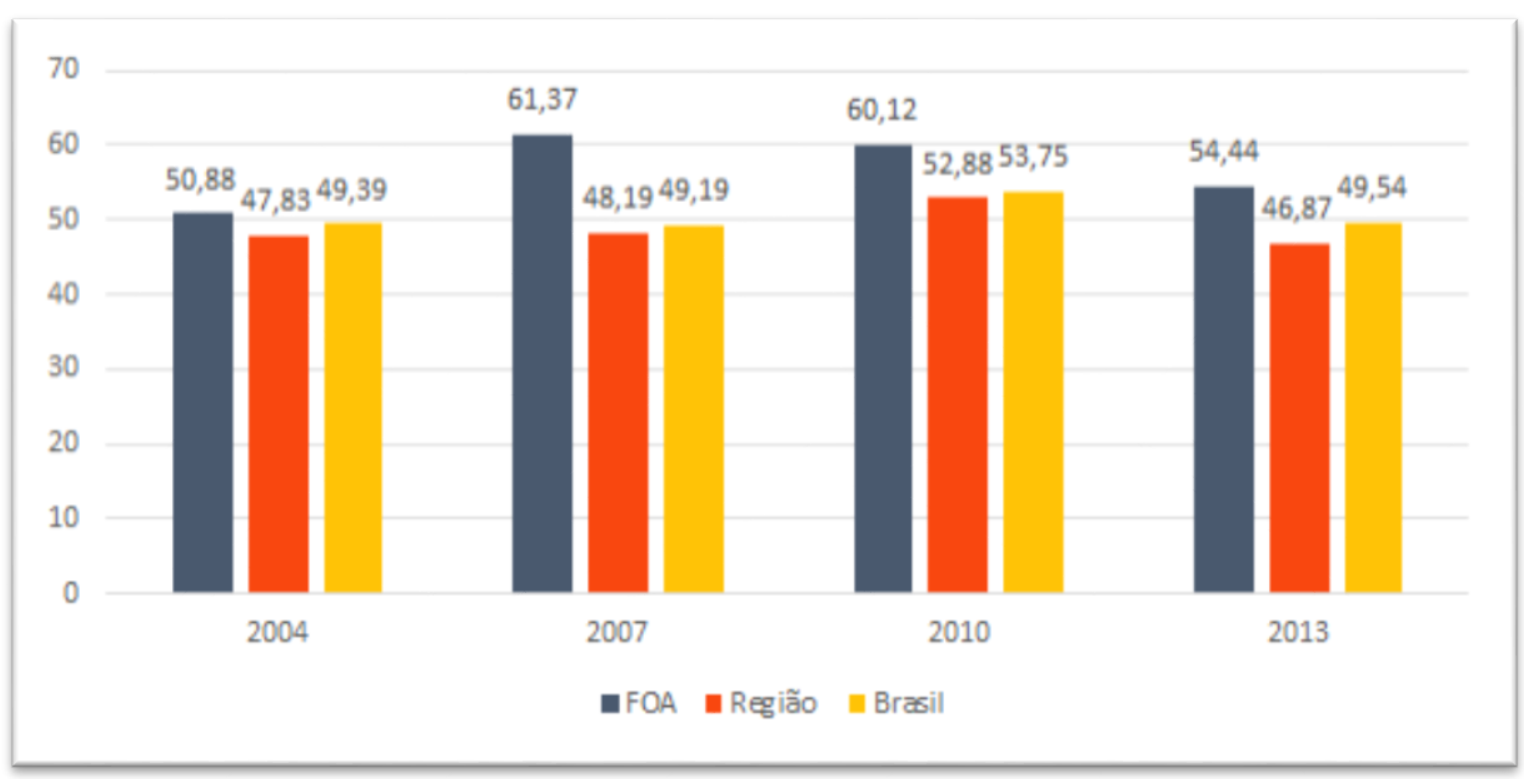

Gráfico 1. Média dos acertos (\%) da Unesp/FOA nas edições do Enade avaliadas comparados com os resultados da Região e Brasil. 


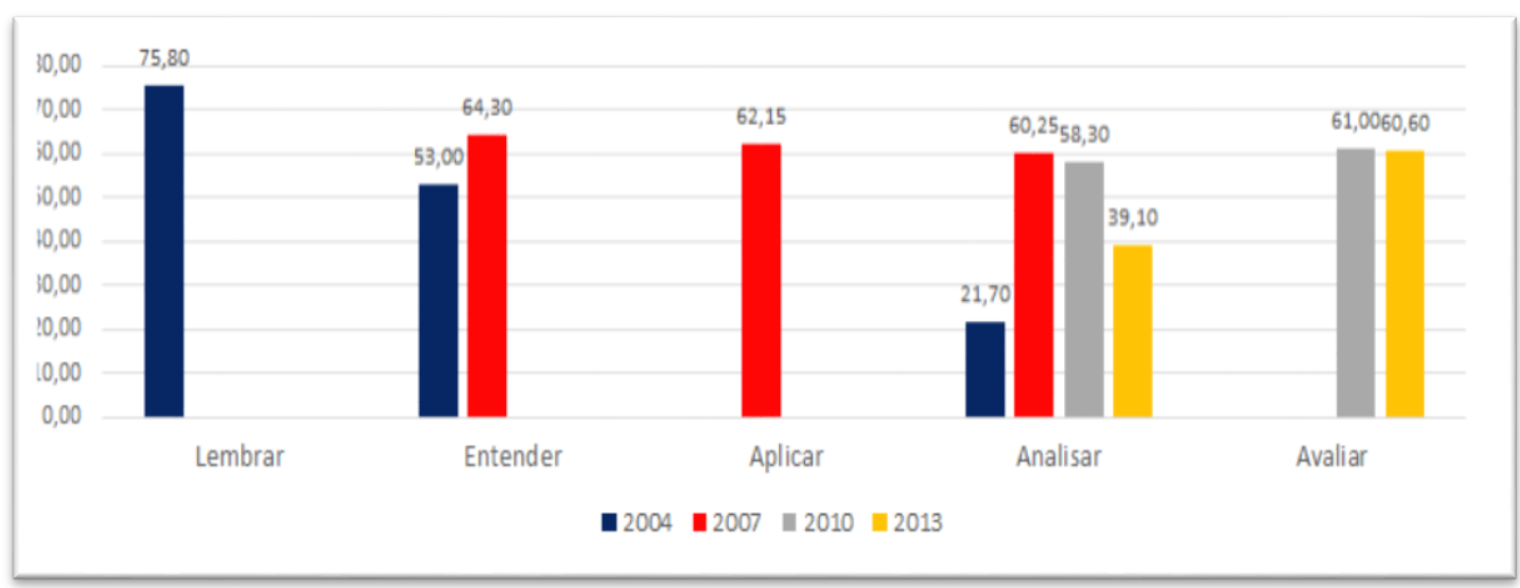

Gráfico 2. Demandas cognitivas avaliadas nas edições do Enade de Odontologia e desempenho (\%) dos estudantes da Unesp/FOA.

A dimensão "Lembrar" relaciona-se a reconhecer e reproduzir ideias e conteúdos. Reconhecer requer distinguir e selecionar uma determinada informação e reproduzir ou recordar está mais relacionado à busca por uma informação relevante memorizada ${ }^{7}$. Observou-se que esta dimensão foi verificada somente no exame de 2004.

O objetivo principal dessa dimensão é fazer com que o estudante memorize, de forma ordenada, o conhecimento a que foi apresentado em sala de aula. No dia a dia, para introduzir conteúdo didático, o professor pode inclusive recorrer a assuntos do cotidiano dos estudantes presentes na mídia, a fim de ativar na memória uma situação específica que os ajude a relembrar o tópico discutido ${ }^{16}$. Deve-se destacar que não é porque a habilidade mnemônica é dimensão primária da aprendizagem que ele se torna necessariamente de fácil obtenção. Nos exames avaliados foi a habilidade cognitiva com maior incidência de acertos entre os estudantes.

A dimensão "Entender" apresentou questões nos exames de 2004 e 2007. A primeira edição apresentou duas questões para esta habilidade intelectual e com média de acerto de $53,00 \%$ dos concluintes que realizaram esta avaliação. O exame de 2007 apresentou uma questão com maior percentual de acerto dos estudantes $(64,30 \%)$ (gráfico 2).

Entender refere-se a estabelecer uma conexão entre o novo e o conhecimento previamente adquirido. A informação é entendida quando $\mathrm{o}$ aprendiz consegue reproduzi-la com suas "próprias palavras"7.

Esta dimensão representa o conjunto de habilidades mais enfatizadas na educação. Compreender significa ter capacidade para entender o conteúdo de uma forma verbal, escrita ou simbólica ${ }^{16}$. Ao todo, foram três questões presentes nas quatro edições do Enade que foram analisados, com uma média de desempenho por parte dos estudantes concluintes de 58,70\%.

A dimensão "Aplicar" foi verificada em uma questão do Enade de 2004 e duas questões em 2007, com $62,15 \%$ de acerto. Vale destacar que a questão 12 foi anulada pelos organizadores por não atenderem aos objetivos do enunciado ${ }^{12}$.

Aplicar relaciona-se a executar ou usar um procedimento em uma situação específica e pode também abordar a aplicação de um conhecimento em uma situação nova ${ }^{7}$.

A dimensão "Aplicar" requer que o estudante seja desafiado com uma situação inédita. Cabe ao docente apresentar um exercício novo e ao discente utilizar os ensinamentos para 
solucioná-lo, como aplicar regras, métodos, modelos, conceitos, princípios, leis e teorias ${ }^{16}$. Por tal complexidade, esta habilidade intelectual apresentou a menor incidência de acertos entre as três primeiras dimensões da Taxonomia dos Objetivos Educacionais.

A dimensão "Analisar" apresentou uma redução na incidência nas edições do Enade estudadas, partindo de quatro questões nas edições de 2007 e 2010 para duas questões na edição de 2013. Os conteúdos abordados foram análise socioepidemiológica dos problemas de saúde bucal da comunidade, elaboração e execução de projetos, programas e outros sistemas de ação coletiva ou de saúde pública visando à promoção, o reestabelecimento e o controle da saúde bucal e gestão administrativooperacional (incluída organização de serviços, gerenciamento e administração em saúde pública, vigilância sanitária, controle das doenças e educação em saúde pública). Duas das oito questões, no entanto, foram anuladas pelos organizadores após a aplicação das provas, sendo esta a questão objetiva 34 e discursiva 40 do exame de 2010 (quadro 1).

O desempenho dos estudantes concluintes decresceu ao logo dos exames para a dimensão “Analisar”. Nas edições de 2007 e 2010, o índice de acerto foi de $60,25 \%$ e $58,30 \%$, respectivamente e, em 2013, verificou-se um desempenho de $39,10 \%$. A média do aproveitamento, portanto, foi de $44,84 \%$ por parte dos que realizaram o exame (gráfico 2).

A dimensão "Analisar" refere-se a dividir a informação em partes relevantes e irrelevantes, importantes e menos importantes e entender a inter-relação existente entre as partes ${ }^{7}$.

Sendo assim, "Analisar" não se destina somente a compreender. Do ponto de vista didático, esta habilidade intelectual se caracteriza pelo fato de agregar a capacidade de distinguir se determinados conteúdos são ou não relevantes em uma comunicação, relacionar ideias, perceber a necessidade de reformulações, enxergar implicações ocultas e as técnicas e recursos utilizados pelo autor, entre outros ${ }^{16}$.

Com o aumento do número de questões desta dimensão, "Analisar" passou a representar $39,29 \%$ de todas as questões do Exame Nacional de Desempenho dos Estudantes nas edições avaliadas. Nos Enade de 2007 e 2010, a dimensão ocupou $36,36 \%$ do total de questões específicas do exame.

Uma vez que a introdução de modificações em estruturas curriculares requer a aplicação de processos padronizados de avaliação, pois o desempenho dos alunos (nos domínios cognitivo, afetivo e psicomotor) é indicador da eficiência do currículo e/ou dos métodos de ensino ${ }^{17}$, faz-se necessário estimular os discentes para tal habilidade cognitiva que apresentou resultados em declínio.

Das dez questões da dimensão "Avaliar", apenas uma foi anulada e refere-se a questão 25 do Enade de $2013^{15}$. Foram quatro questões $(40,00 \%)$ na edição de 2010 e seis questões $(60,00 \%)$ na última edição avaliada do desempenho dos estudantes concluintes.

O desempenho dos egressos foi de $61,00 \%$ na edição de 2010, com acerto variando de $43,10 \%$ a $81,40 \%$. Para a edição de 2013 , a média foi de $60,60 \%$ com variação de $32,30 \%$ a $79,20 \%$ de acertos dos egressos da Faculdade de Odontologia de Araçatuba. Os conteúdos abordados também foram análise socioepidemiológica dos problemas de saúde bucal da comunidade, elaboração e execução de projetos, programas e outros sistemas de ação coletiva ou de saúde pública visando à promoção, o reestabelecimento e o controle da saúde bucal e gestão administrativo-operacional (gráfico 2).

A dimensão "Avaliar" ocupou espaço significativo nas edições do Enade em Odontologia $(35,71 \%)$ e especialmente no último 
exame $(20,00 \%)$ da prova de conhecimentos específicos para os conteúdos de Saúde Coletiva.

Esta dimensão relaciona-se a realizar julgamentos com base em critérios e padrões qualitativos e quantitativos ou de eficiência e eficácia ${ }^{7}$.

A dimensão se encontra em um estágio avançado do domínio cognitivo porque engloba todos os comportamentos observados anteriormente: "Lembrar, Entender, Aplicar e Analisar". Um dos maiores desafios do sistema educacional moderno é mesmo o processo avaliativo $^{16}$.

Acredita-se que o desempenho dos estudantes concluintes foi satisfatório para a dimensão "Avaliar" pelo fato de a Odontologia ser uma ciência multidisciplinar e tal habilidade intelectual compor o Projeto Pedagógico de Curso da Faculdade de Odontologia de Araçatuba, em especial nos conteúdos da área de Saúde Coletiva.

Desenvolver a capacidade de abstração e utilização de um conhecimento específico de forma multidisciplinar é um processo que deve ser bem planejado, definido e organizadamente estimulado durante o período de formação (graduação), levando-se em consideração os estilos de aprendizagem ${ }^{18}$.

O nível de cognição criar, não foi encontrado em nenhuma questão de Saúde Coletiva dos Enade avaliados, possivelmente em função da limitação do tempo e da quantidade de questões. Nesta dimensão, o discente deve desenvolver ideias novas e originais por meio da percepção da interdisciplinaridade e interdependência de conceitos ${ }^{19}$. E esta é uma dimensão pouco percebida na formação de um cirurgiãodentista.

Processo cognitivo pode ser entendido como o meio pelo qual o conhecimento é adquirido ou construído e usado para resolver problemas diários e eventuais ${ }^{8}$.
Atualmente, alguns autores ${ }^{20,21}$, consideram processos cognitivos de níveis baixos as dimensões "Lembrar e Entender", e níveis altos correspondem aos processos mais complexos "Aplicar, Analisar, Avaliar e Criar".

Estudo de Moimaz et al. ${ }^{22}$, comprova a eficiência da extensão universitária como agente fomentador dos domínios afetivo, cognitivo e psicomotor. O programa de extensão revelou ser um importante agente para o processo individual e coletivo de formação acadêmica, que não se limita ao tradicional, uma vez que, contribui para uma formação em saúde mais humanista, generalista e preparado para atuar frente à situação de saúde de nosso país.

Outro ponto a ser observado é a importância de se manter o equilíbrio entre questões de alta e baixa demanda cognitiva, assegurando um processo de formação plena, e que pode apresentar melhor desempenho dos alunos a partir da utilização da taxonomia dos objetivos cognitivos de Bloom nos Projetos Pedagógicos de Cursos de Odontologia.

Um ponto central do Exame Nacional de Desempenho dos Estudantes é verificar se e de que maneira a IES está contribuindo para a formação do estudante, não apenas na parte referente à assimilação de conhecimentos sobre determinados objetos, mas, particularmente, o que o estudante agrega na passagem por aquela instituição específica, com suas características peculiares e localizada em um determinado contexto, e com um objetivo maior de desenvolver habilidades acadêmicas e competências profissionais ${ }^{23}$.

\section{CONCLUSÕES}

Os objetivos educacionais "Analisar" e "Avaliar" foram mais prevalentes nas edições do Exame Nacional de Desempenho dos Estudantes e as questões classificadas nestas dimensões de maior nível cognitivo apresentaram menor taxa 
de acertos, quando comparadas às dimensões "Lembrar", "Entender" e "Aplicar".

Os resultados ainda indicam que nos anos de 2010 e 2013, houve um aumento do número de questões pertencentes a dimensão de alto nível cognitivo, podendo indicar uma aproximação do pressuposto teórico previsto nas Diretrizes Curriculares Nacionais de Odontologia.

\section{ABSTRACT \\ Enade in Dentistry: analysis and reflections using Revised Bloom Taxonomy}

The objective of this study was to analyze the Collective Health $(\mathrm{CH})$ questions in the Dentistry National Student Examination editions from 2004 to 2013, using the Revised Bloom Taxonomy: "Remember, Understand, Apply, Analyze, Evaluate, Create" and the undergraduate's performance of the São Paulo State University (Unesp), School of Dentistry, Araçatuba in this exam. A documentary research was carried out, analyzing the evaluations of 2004, 2007, 2010, 2013.To identify the $\mathrm{CH}$ questions presented in the exams, it was used the knowledge objects described in the Article 86 of the Consolidation of Rules for Procedures in the Boards of Dentistry and then ranked according to the Revised Bloom Taxonomy (RBT): "Remember, Understand, To apply, Analyze, To evaluate, Create". It was also identified the performance of the concluding undergraduates of UNESP/FOA. We evaluated 28 questions, of which 23 were objective and five were discursive. Considering the Enade editions, "Remember", "Understand" and "Apply" dimensions of the RTB were verified in seven questions and the mean of undergraduates successful for these dimensions was $65.53 \%$. The "Analyze" dimension presented one question in 2004, four questions in 2007 and 2010 editions and two questions in the 2013 edition, and the mean which represents the successful of the undergraduates was $44.84 \%$. Considering 10 questions of the "Evaluate" dimension, the performance was $61.00 \%$ in the 2010 edition, with a score varying from $43.10 \%$ to $81.40 \%$, and for the 2013 edition, the mean was $60.60 \%$ varying from $32.30 \%$ to $79.20 \%$. The "Analyze" and "Evaluate" educational objectives were more prevalent in the Dentistry National Student Examination, and the questions classified in these higher cognitive dimensions presented a lower hit rate when compared to the "Remember", "Understand", and "To apply".

Descriptors: Educational Measurement. Classification. Teaching. Dentistry.

\section{REFERÊNCIAS}

1. Moimaz SAS, Amaral MA, Garbin CAS. Enade: uma análise quanti-qualitativa dos exames nacionais de Odontologia. Rev ABENO. 2017; 17(1): 97-108.

2. Brasil. Lei $n^{\circ} .9 .131$, de 24 de novembro de 1995. Altera dispositivos da Lei $n^{\circ} .4 .024$, de 20 de dezembro de 1961 e dá outras providências. Diário Oficial [da República Federativa do Brasil], Brasília: DF, 25 nov.1995. [Acesso em 30 jun. 2017]. Disponível em: http://www.planalto.gov.br/ ccivil_03/leis/L9131.htm

3. Paiva GS. Avaliação do desempenho dos estudantes da educação superior: a questão da equidade e obrigatoriedade no Provão e Enade. Ensaio: Aval Pol Públ Educ. 2008; 16(58):31-46.

4. Brasil. Lei $n^{\circ} .10 .861$, de 14 de abril de 2004. Institui o Plano Nacional de Avaliação da Educação Superior e dá outras providências. Diário Oficial [da República Federativa do Brasil], Brasília: DF, n. 72, 15 abr. 2004. [Acesso em 30 jun. 2017]. Disponível em: http://www.planalto.gov.br/ccivil_03/_ato20 04-2006/2004/lei/110.861.htm

5. Brasil. Lei ${ }^{\circ}$. 13.005, de 25 de junho de 2014. Institui o Plano Nacional de Educação (PNE) e dá outras providências. Diário Oficial [da República Federativa do Brasil], Brasília: DF, Seção 1 - Edição Extra, 26 jun. 2014. [Acesso em 30 jun. 2017]. Disponível em: http://www.planalto.gov.br/ccivil_03/_ato20 11-2014/2014/lei/113005.htm

6. Bloom BS, Hastings JT, Madaus GF. Handbook on formative and sommative evaluation of student learning. New York: McGraw-Hill, 1971. 
7. Ferraz APCM, Belhot RV. Taxonomia de Bloom: revisão teórica e apresentação das adequações do instrumento para definição de objetivos instrucionais. Gest Prod. 2010; 17(2):421-31.

8. Anderson LW, Krathwohl DR, Bloom BS. A taxonomy for learning, teaching, and assessing: a revision of Bloom's taxonomy of educational objectives. New York: Longman, 2001.

9. Tikkanen G, Aksela M. Analysis of finnish chemistry matriculation examination questions according to cognitive complexity. NorDiNa. 2012; 8(3): 258-68.

10. INEP. Instituto Nacional de Estudos e Pesquisas Educacionais Anísio Teixeira. Exame Nacional de Desempenho dos Estudantes - Enade. [Acesso em 20 set. 2016]. Disponível em: http://portal.inep.gov.br/ enade/provas-e-gabaritos.

11. Conselho Federal de Odontologia. Resolução 63, de 30 de junho de 2005. Institui a Consolidação das Normas para Procedimentos nos Conselhos de Odontologia.

12. INEP. Instituto Nacional de Estudos e Pesquisas Educacionais Anísio Teixeira. Exame Nacional de Desempenho dos Estudantes - Relatório de Curso 2005. [Acesso em 10 nov. 2016]. Disponível em: http://enadeies.inep.gov.br/enadeIes/boletim Desempenho/.

13. INEP. Instituto Nacional de Estudos e Pesquisas Educacionais Anísio Teixeira. Exame Nacional de Desempenho dos Estudantes - Relatório de Curso 2008. [Acesso em 10 nov. 2016]. Disponível em: http://enadeies.inep.gov.br/enadeIes/boletim Desempenho/.

14. INEP. Instituto Nacional de Estudos e Pesquisas Educacionais Anísio Teixeira. Exame Nacional de Desempenho dos Estudantes - Relatório de Curso 2011. [Acesso em 10 nov. 2016]. Disponível em: http://enadeies.inep.gov.br/enadeIes/boletim Desempenho/.

15. INEP. Instituto Nacional de Estudos e Pesquisas Educacionais Anísio Teixeira.
Exame Nacional de Desempenho dos Estudantes - Relatório de Curso 2014. [Acesso em 10 nov. 2016]. Disponível em: http://enadeies.inep.gov.br/enadeIes/boletim Desempenho/.

16. Nicolini AM, Andrade ROB. Padrão Enade: análise, reflexões e proposições à luz da Taxonomia de Bloom. São Paulo: Atlas, 2015.

17. Piccinato CE, Figueiredo JFC, Troncon LEA, Peres LC, Cianflone ARL, Colares MFA et al. Análise do desempenho dos formandos em relação a objetivos educacionais da Faculdade de Medicina de Ribeirão Preto, Universidade de São Paulo, em duas estruturas curriculares. Rev Assoc Méd Bras. 2004; 50(1):68-73.

18. Belhot RV, Freitas AA, Vasconcellos DD. Requisitos profissionais do estudante de engenharia de produção: uma visão através dos estilos de aprendizagem. Rev GEPROS. 2006; 1(2):125-35.

19. Silva VF, Martins MI. Análise de questões de física do Enem pela taxonomia de bloom revisada. Rev Ensaio. 2014; 16(3):189-202.

20. Crowe A, Dirks C, Wenderoth MP. Biology in bloom: implementing Bloom's taxonomy to enhance student learning in biology. CBE Life Sci Educ. 2008; 7(4): 368-81.

21. Zoller U. Are lecture and learning compatible? Maybe for LOCS: Unlikely for HOCS. J Chem Educ. 1993; 70(3):195.

22. Moimaz SAS, Bordin D, Gomes AMP, Fadel CB, Garbin CAS, Saliba NA. Extensão universitária da ótica de acadêmicos: o agente fomentador das Diretrizes Curriculares Nacionais. Rev ABENO. 2015; 15(4):45-54.

23. Brito, MRF. Enade 2005: Perfil, desempenho e razão da opção dos estudantes pelas Licenciaturas. Avaliação (Campinas). 2007; 12(3): 401-43.

\section{Correspondência para}

Profa. Dra. Suzely Adas Saliba Moimaz

E-mail: sasaliba@foa.unesp.br

Programa de Pós-Graduação em Odontologia Preventiva e Social

Rua José Bonifácio, 1193 Vila Mendonça

16015-050 Araçatuba/SP 